\title{
Hashimoto's Thyroiditis
}

\author{
Arvin Parvathaneni, Daniel Fischman and Pramil Cheriyath \\ Pinnacle Health System-Harrisburg Hospital \\ Harrisburg, Pennsylvania, \\ USA
}

\section{Introduction}

Hashimoto's thyroiditis is a common autoimmune disorder, which causes significant morbidity. Its pathophysiological hallmark is lymphocytic infiltration of thyroid follicles resulting in autoimmune glandular destruction. Various studies have successfully outlined the genetic and environmental factors responsible for the causation of the disease. In this chapter we will discuss our current understanding of these factors and delineate how Hashimoto's thyroiditis serves as a paradigm not just for disease of the thyroid gland, but also for autoimmune disease in the human body. Our focus is on the varying presentations of the disease and the relationship between Hashimoto's thyroiditis and other autoimmune diseases frequently associated with it. The etiological factors and the pathophysiological changes which lead to the development of disease are discussed. Common diagnostic modalities are described, and the need for correlation between the various available diagnostic tests is explained. Various treatment strategies and the appropriate choice for different forms of presentation are discussed. Hashimoto's encephalopathy, a rare complication, will be addressed separately as its unusual presentation often results in misdiagnosis of the underlying pathology.

\section{Background}

Hashimoto's thyroiditis was first described in 1912 by Dr. Hakuru Hashimoto. Based on the histological findings, Hashimoto originally used the term "Struma Lymphomatosa." Over the years, this disease has been called by several names including lymphocytic thyroiditis, autoimmune thyroiditis, chronic thyroiditis, and lymph adenoid goiter. The debate about the relationship between Hashimoto's thyroiditis and Graves' disease has been ongoing for many decades as they differ in clinical and immunological presentation. However, Hashimoto's thyroiditis and Graves' disease, which depict the two extremes of the clinical spectrum, are now included in a common entity called autoimmune thyroid disease. It is now believed that they share a common autoimmune pathology and are believed to be triggered by multiple genetic and environmental factors. Hashimoto's thyroiditis was initially perceived as an uncommon disease and most cases were incidentally diagnosed through histopathological examination of the thyroid gland after thyroidectomy. The advent of newer diagnostic modalities with increased diagnostic sensitivity made it possible to 
unveil more cases of Hashimoto's thyroiditis. With the increasing number of cases, the association of Hashimoto's thyroiditis with other autoimmune diseases is being studied extensively. Type 1 diabetes, multiple sclerosis, rheumatoid arthritis, celiac disease, vitiligo, and chronic urticaria have all been reported to be frequently associated with Hashimoto's thyroiditis.

\section{Incidence and distribution of the disease}

Hashimoto's thyroiditis is about 15-20 times more common in women than in men and frequently involves people between the ages of 30 and 50 years of age. Determining the exact incidence and prevalence rates for Hashimoto's thyroiditis has been difficult due to variable expression of this disease. Some studies estimate that the current prevalence rate in the United States ranges between $0.3 \%-1.2 \%$ (Staii et al., 2010). Other studies estimate the prevalence among the general population to be approximately $2 \%$ (Wang et al., 1997). When attempts have been made to characterize the prevalence prospectively, with the aid of organized programs of ultrasound guided biopsy, the prevalence described has been at least $5 \%$. It should be noted that studies employing the diagnostic modality of ultrasound guided biopsy have recorded prevalence rates higher than studies using other investigative modalities (Staii et al., 2010). The National Health and Nutrition Evaluation Study-3 (NHANES-3) study has shown the prevalence of subclinical and clinical hypothyroidism to be $4.6 \%$ and $0.3 \%$, respectively, in the United States (Hollowell et al., 2002). The Whickham survey, an epidemiological study conducted in the United States, has revealed the prevalence of hypothyroidism to be $1.5 \%$ in females and less than $0.1 \%$ in males (Tunbridge et al., 1997). During the past few decades there has been a reported increase in the incidence of Hashimoto's thyroiditis, which could be attributed to newer diagnostic modalities such as needle biopsies and serological tests, and their increased sensitivity when compared to the older methods. (McConahey et al., 1962). Studies about age-specific incidence rates of Hashimoto's thyroiditis indicate the existence of a random distribution in both men and women and have shown an initial lag in the first few years of their life followed by a constant rate after this (Volpe et al., 1973). A few studies have suggested a slight increase in the prevalence of autoimmune thyroiditis in adolescent girls following use of iodized food products ingested to prevent iodine deficiency (Zois et al., 2003).

\section{Etiology}

The etiology of Hashimoto's thyroiditis is considered to be multifactorial, involving the interplay of various environmental and genetic factors. Studies conducted on the genetic associations of Hashimoto's thyroiditis have shown that the human leukocyte antigen (HLA) region, which plays a major role in other autoimmune disorders, is associated with development of Hashimoto's thyroiditis (Fisher, G.F., 2000). The association of Hashimoto's thyroiditis with various other autoimmune diseases has further reinforced the probable involvement of genetic factors in the etiology. The major histocompatability complex (MHC), cytotoxic T-lymphocyte association (CTLA-4) and the human leukocyte antigen (HLA) are the genetic factors which are purported to play a major role in the pathogenesis. The selection of thyroid cells in the thymus and presentation of antigens in the periphery are modulated by, the human MHC analog, HLA. The sensitivity and specificity of the affinity to bind the peptides and recognize T-cells is determined largely by the genetic 
polymorphisms exhibited by the MHC molecule. The possible polymorphisms within the MHC molecules play a pivotal role in the predisposition to autoimmune disease (Gebe et al., 2002). The association between the genetics of Hashimoto's thyroiditis and HLA gene loci has been investigated by serotyping the HLA, and deoxyribonucleic acid (DNA) typing the sequence- specific oligonucleotides. Different subsets of HLA genes have been found to show varying degree of associations with Hashimoto's thyroiditis in different races. The HLA class 1 and class 2 genes both showed association with Hashimoto's thyroiditis in Asian populations, while only HLA class 1 demonstrated the association in Caucasians. $(\mathrm{Wu}$ et al., 1994). No significant associations have been found between Hashimoto's thyroiditis and HLA class 3 or non-HLA genes of the HLA region (Hunt et al., 2001). An association between CTLA-4 and Hashimoto's thyroiditis has been noted in significant number of cases (Einarsdottir et al., 2003). CTLA-4 plays a vital role in upholding immunological self tolerance in the body and its down regulation is believed to be the initiating step for the pathogenesis of Hashimoto's thyroiditis as well as other autoimmune disorders such as Graves' disease (Chistiakov \& Turakulov, 2003).

In addition to the genetic factors numerous external factors also play a vital role in the etiology of the disease, preferentially affecting genetically predisposed individuals. The common environmental factors which act as triggers to initiate the insult on thyroid tissue include infections, cytokine therapy, selenium and iodine intake. Epidemiological studies and animal models have shown that among the factors that initiate the process, iodine appears to be the most significant (Boukis et al., 1983). Some studies have established smoking as an important risk factor for the causation of hypothyroidism in patients with Hashimoto's thyroiditis (Fukata et al., 1996).

\section{Pathogenesis}

The pathogenesis of Hashimoto's thyroiditis is a complex multistep process which involves various genetic, environmental and immunological factors ( Figure 1). In a nut shell, loss of immune tolerance to normal thyroid cells leads to production of antibodies directed against thyroid tissue, which causes the destruction of the thyroid gland. The initial inflammatory changes in the disease process are triggered when genetically predisposed individuals are exposed to the above mentioned environmental factors. The major histocompatability complex (MHC) class 2 antigen presenting cells, which include dendritic cells and macrophages, invade the thyroid gland after the initial inflammatory process. These cells present the autoantigen components of the thyroid gland to the immune system for processing. Among the myriad of potential auto-antigens, thyroglobulin, the main protein produced in thyroid tissue, is believed to play a central role in the pathogenesis of this disease (Champion et al., 1991). The thyroglobulin protein has been reported to have approximately 40 different types of epitopes, which play a vital role in the pathogenesis of the disease (Male et al., 1985). In contrast to the epitope recognition pattern of normal individuals, the epitope recognition pattern of the antibodies in autoimmune thyroid disease is altered triggering immune and inflammatory processes (Dietrich et al., 1991). Thyroid peroxidase, an enzyme that catalyzes the oxidation of iodine, also plays a significant role as an autoantigen in the disease pathogenesis. Moreover, 180 different types of thyroid peroxidase antibodies have been identified, thus far. Studies have confirmed that even though antibodies against thyrotropin receptor and sodium iodide symporter have 
been detected in patients with autoimmune thyroid disease, they do not play a significant role in the pathogenesis of this condition.

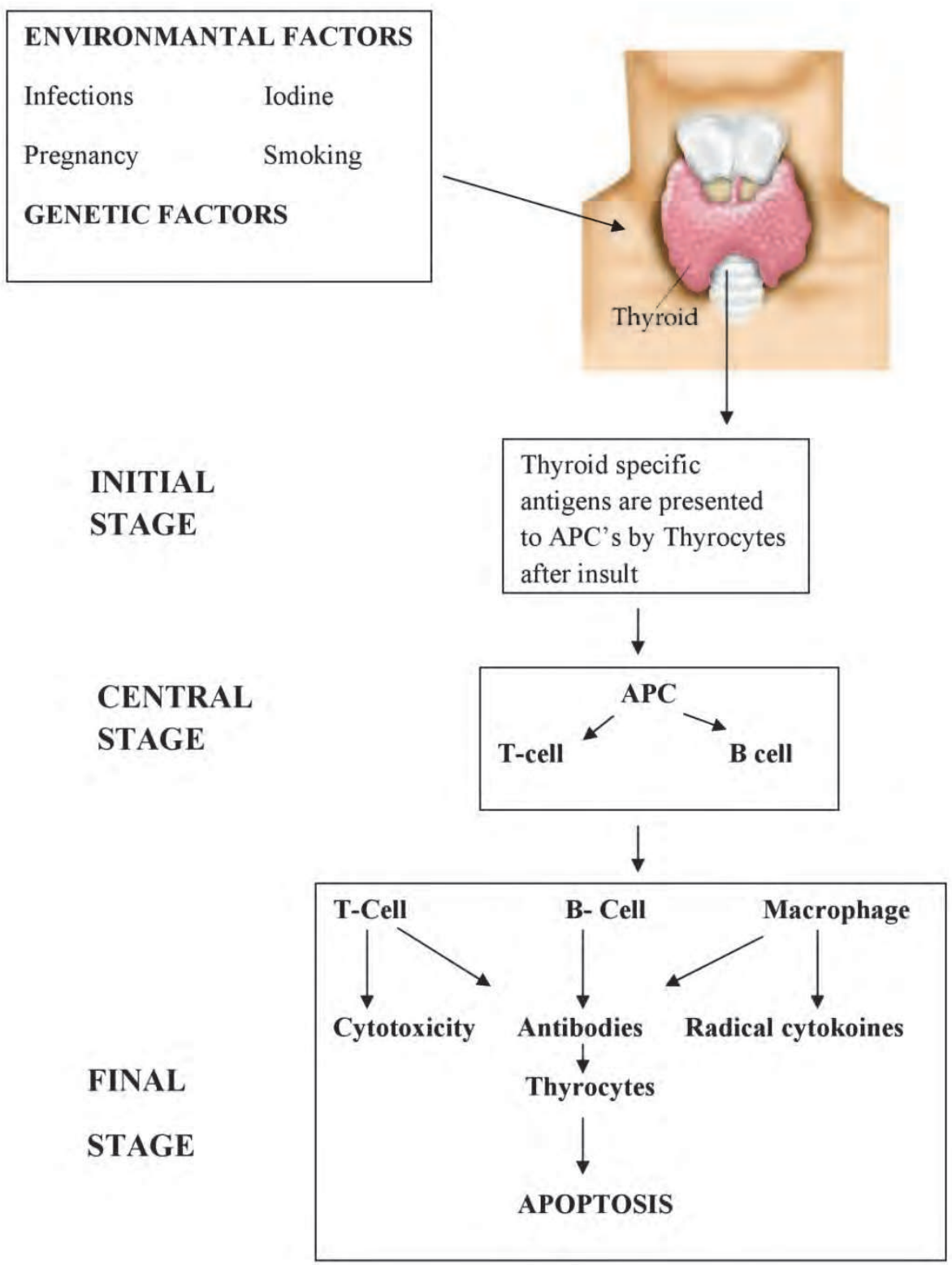

Legend: $\mathrm{APC}=$ Antigen Presenting Cell

Fig. 1. A schematic presentation of etiopathogenesis of Hashimoto's thyroiditis. (Casselman, W, G., 1996). 
The major step in the pathogenesis is the formation of autoreactive cells directed against the thyroid gland, which could result from defects in central tolerance or defects in the peripheral tolerance. Loss of immune tolerance has been associated with genetically determined immune defects or with the lack of regulatory T-cells which impose the suppressive function (Martin, 1992). This is followed by formation, clonal expansion, and maturation of self-reactive T-lymphocytes and B-lymphocytes in the draining lymph nodes. This step is then followed by a central phase of autoimmunity, characterized by uncontrolled production of self-reactive cells and autoantibodies in response to the presented antigens. This process initially occurs in the lymph nodes but as the disease progresses the production process shifts to the thyroid gland where the development of lymphoid tissue follows. The stimulated B-lymphocytes produce antithyroglobulin (TGAB) and antithyroid peroxidase (ATPO) antibodies which are directed against thyroid cells. The autoreactive T-cells, which are produced in the disease process, infiltrate the thyroid gland and mediate destruction through cytotoxicity with the aid of $C D+8$ cells. The macrophages which are stimulated in this process produce numerous cytokines which, along with antibodies, initiate the process of tissue destruction via apoptosis.

As a final step in the process, caspases, which are self-activated through proteolytic cleavage, induce enzymes which are directly involved in the destruction of thyroid gland. In a normal thyroid gland, the production of new cells and the destruction of old cells are tightly regulated so that a constant proportion of functioning cells is always present. During the course of the disease, the control over destruction of cells in the thyroid gland is lost. Genetic susceptibility is one of the factors that plays a vital role in deregulation of the regular destructive mechanisms in the thyroid gland. Several other triggers which have an influence on the expression of $\mathrm{Bcl}-2$, the apoptosis inhibitor, or Fasl membrane ligand also are crucial in the initiation of the apoptosis process (Giordano et al., 2001). Thyroid cells in tissue affected with Hashimoto's thyroiditis, when compared to normal thyroid cells, are capable of producing more Fasl proteins leading to an increased tempo of apoptosis (Limachi \& Basso, 2002). The severity of the disease and the clinical outcome are determined by the rate at which apoptosis occurs in the thyroid gland. Expression of these proteins has direct correlation to the severity of the disease and as the rate of apoptosis increases, the mass of hormonally-active thyroid tissue decreases resulting in diminished production of thyroid hormones and more significant disease manifestations.

\section{Signs and symptoms}

Thyroid hormone is capable of influencing the function of every cell in the body. The basic function of thyroid hormone is to increase the basal metabolic rate of the body. The symptoms of Hashimoto's thyroiditis are predominantly due to decreased production of thyroid hormone, which occurs as a result of destruction of thyroid tissue, ultimately leading to decreased metabolism. Indeed, most symptoms are not manifested in the early stages of the disease; as the disease advances and the degree of hypothyroidism increases, the symptoms become more evident. The decreased production of thyroid hormone adversely affects various major organ systems. Dysfunction of the cardiovascular system is manifested as bradycardia, while nervous system dysfunction manifests as slowed speech and delayed reflexes. Gastrointestinal symptoms include constipation, increased bile reflux 
and ascites. When the metabolic rate drops to a critical level, a life threatening emergency called myxedema coma occurs. Myxedema is usually characterized by hypothermia, hypoglycemia, altered sensorium and severe bradycardia. In severely hypothyroid Individuals, triggers such as stress, infection, surgery and traumatic injuries may also predispose to the development of myxedema.

In contrast to hypothyroid patients, patients in a euthyroid state do not experience any symptoms or exhibit any signs of the disease, and in most cases the diagnosis is incidental. Moreover, some individuals may not present with any clinical features except an enlarged thyroid gland and the diagnosis is made by investigating the goiter. The goiter, by itself, can cause cosmetic disfigurement in its initial stages and as its size increases, it can lead to pressure symptoms including pain in the neck, dysphagia, and dyspnea in some cases. Furthermore, a rapid growth in the goiter is sometimes noted, which should arouse suspicion for a tumor. Tumors of the thyroid gland, which sometimes arise in the background of Hashimoto's thyroiditis, usually manifest as solitary or multiple nodules typically discovered incidentally during a regular physical examination. In addition to the previously noted symptoms, accumulation of the matrix proteins, such as metalloproteases, might lead to swelling of the extremities and face. Though extremely rare in children, Hashimoto's thyroiditis can lead to detrimental effects on growth and physical maturation. Moreover, short stature and mental retardation are the features which are most commonly observed in children suffering from Hashimoto's thyroiditis.

In addition to the symptoms of hypothyroidism, people suffering from Hashimoto's thyroiditis sometimes experience symptoms due to other autoimmune diseases. Muscle pain is present in $25.5 \%$ of patients with Hashimoto's thyroiditis. Rheumatic manifestations in autoiummune thyroiditis are reported to be ten times more frequent when compared to nonautoimmune thyroiditis. (Becker et al., 1963). Furthermore, the initial presentation sometimes can be very subtle. For instance, occasionally, irritatability, depression, confusion, and fatigue have been reported as initial complaints in patients later diagnosed with Hashimoto's thyroiditis (Hall et al., 1982). Unfortunately, in many instances these cases were misdiagnosed as psychiatric disorders before being correctly diagnosed as due to thyroid hormone deficiency.

\section{Clinical course of the disease}

Hashimoto's thyroiditis has a highly variable clinical presentation; patients may either be hypothyroid, euthyroid or hyperthyroid. About $20 \%$ of the patients exhibit signs and symptoms of mild hypothyroidism at the initial presentation. However, the severity of the symptoms increases with the progression of the disease (Gordin et al., 1974). This increase in the severity of symptoms is attributed to gradual destruction of the thyroid gland. Furthermore, as the hypothyroidism worsens, the patient is at increased risk of developing myxedema coma as a result of complete thyroid atrophy (Buchanan \& Harden, 1965). A goiter, usually with gradual enlargement of the gland, may be the sole presentation in some instances. (Tunbridge et al., 1977). The other features which are usually associated with Hashimoto's thyroiditis are not exhibited along with the goiter. 


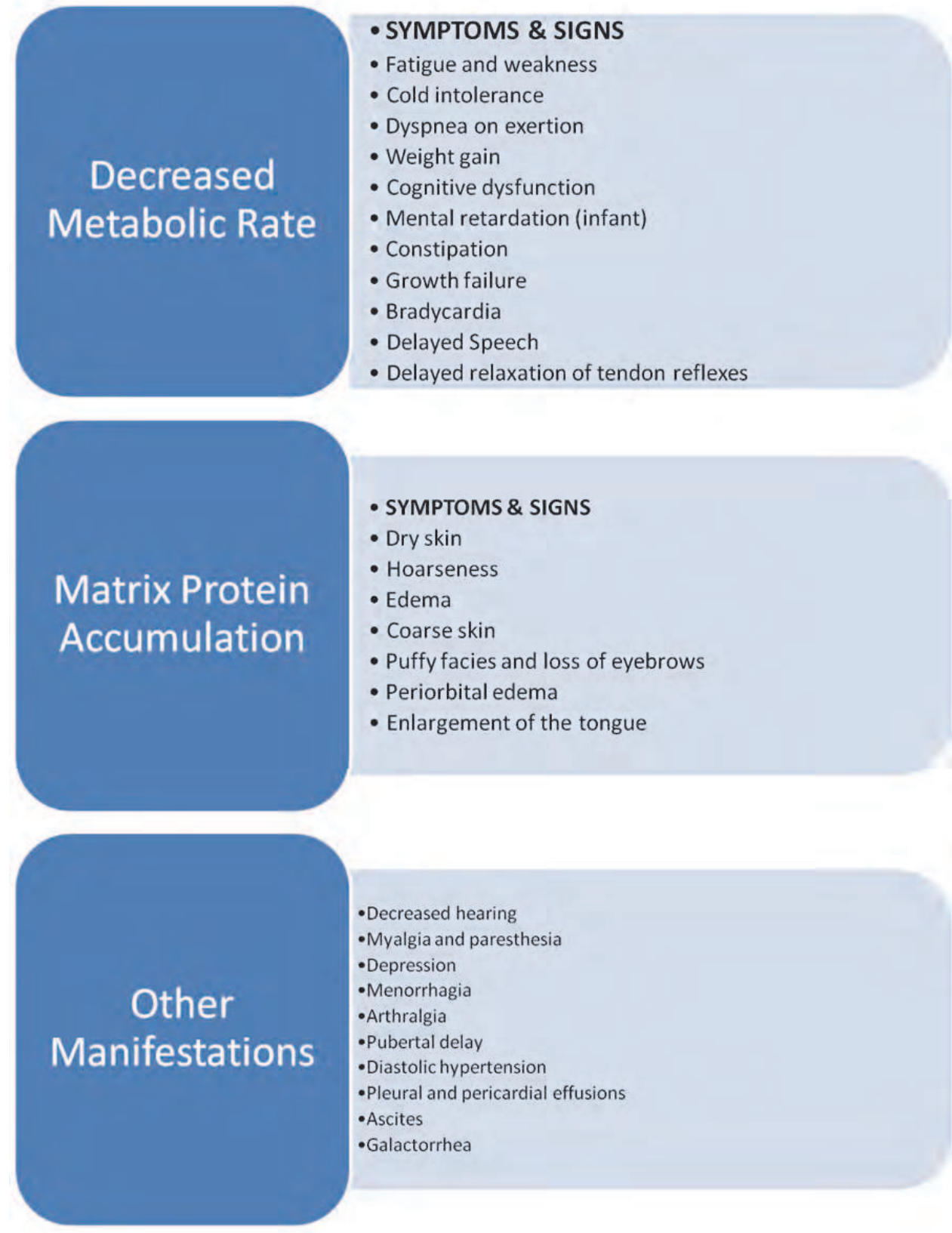

Fig. 2. Symptoms and signs of hypothyroidism based on specific pathophysiology 
Some patients are initially euthyroid and are at risk of developing hypothyroidism as the disease progresses. The concomitant presence of a goiter along with elevated thyroid antibody levels, at presentation, has been found to increase the risk of hypothyroidism (Radetti et al., 2006). Although, for a long time, it was believed that hypothyroidism secondary to Hashimoto's thyroiditis was irreversible, some recent studies have proved otherwise. This assumption is based on observational studies which revealed a decline in titers of thyroid antibodies after the patient was treated with thyroid hormone. Therefore, frequent monitoring of thyroid function has been recommended which would help in accurately assessing the functional status of the thyroid and enable the physician to make necessary changes in the management of the disease (Takasu et al., 1992). Identifying the clinical progression of the disease is important in determining the nature of treatment provided to each individual.

The autoimmune nature of Hashimoto's thyroiditis predisposes patients to concomitant development of additional autoimmune diseases. One such autoimmune disorder is systemic lupus erythematosus. This particular association was reported as early as 1957 (Wilkinson \& Sacker, 1957) and later studies have confirmed this finding (Weetman \& Walport 1987; Pyne \& Isenberg, 2002). An increased incidence of Hashimoto's thyroiditis has been reported in adults suffering from vitiligo; the risk has been assessed to be 2.5 times higher when compared to an age-matched population without Hashimoto's thyroiditis (Kakourou et al., 2005). As per Kakourou et al, annual screening for Hashimoto's thyroiditis is recommended in people suffering with vitiligo. Celiac disease, an autoimmune disorder of the small intestine, is more common in people with autoimmune thyroid disorders when compared to those with other thyroid disorders (Cuoco et al., 1999). Chronic idiopathic urticaria, an autoimmune disorder characterized by bouts of hives, has also been reported to be associated with Hashimoto's thyroiditis although the pathogenesis of chronic urticaria in Hashimoto's thyroiditis is not well understood. Furthermore, chronic idiopathic urticaria has also been reported in euthyroid patients who are seropositive for antithyroid antibodies (Rottem, 2003). In addition, the Ccells in the thyroid gland, which are responsible for the production of calcitonin, and which are involved in the homeostasis of calcium, are damaged by the Hashimoto's thyroiditis disease process. Because of this, patients with Hashimoto's thyroiditis patients have an inherent risk of developing hypocalcemia (Lima et al., 1998). Dyslipedemia has also been reported as one of the complications of Hashimoto's thyroiditis, with thyroid stimulating hormone and free T4 hormone levels being inversely correlated with severity of lipid abnormality (Tagami et al., 2010).

Unlike the clear association between Hashimoto's thyroiditis and other autoimmune diseases, the link between Hashimoto's thyroiditis and cancer is not well delineated. Despite the association first being reported in 1951, the link still remains obscure and is a subject of debate. In investigating various tumor types, lymphoma and papillary carcinoma of thyroid (PTC) are most commonly associated with Hashimoto's thyroiditis. The incidence of thyroid carcinoma in people with Hashimoto's thyroiditis has been reported to be as high as $36.4 \%$ (Pino Rivero et al., 2004). Although a chimeric gene rearrangement has been proposed as the molecular basis for the development of thyroid carcinoma in the presence of Hashimoto's thyroiditis, recent studies have not supported this supposition. Interestingly, no such 
rearrangements were detected in patients diagnosed with papillary thyroid cancer in the background of Hashimoto's thyroiditis, while in patients diagnosed first with PTC, the prevalence of these rearrangements was found to be $33 \%$ (Nikiforova et al., 2002). Unlike with production of chimeric gene products, the tumor protein P63 is believed to play a vital role in the development of PTC in patients with previously established Hashimoto's thyroiditis. Moreover, supporting evidence can be inferred from the absence of these proteins in thyroid tissue devoid of PTC or Hashimoto's thyroiditis (Unger et al., 2003). Similar to PTC, B-cell lymphoma of the thyroid gland has been found to be associated with Hashimoto's thyroiditis. The histological features of this specific lymphoma have been found to be similar to those of mucosa associated lymphoid tumors (Hygek \& Isaacson, 1988).

\section{Diagnosis}

Assessing the metabolic status of the patient and identifying the type of lesion present are of vital importance in making an accurate diagnosis of Hashimoto's thyroiditis. The first step is to assess the thyroid hormone status, which reflects glandular function. Although the presence of goiter alone, without associated hyperthyroid symptoms, is suggestive of Hashimoto's thyroiditis, the presence of a goiter in a hypothyroid patient is considered to be strongly indicative of Hashimoto's thyroiditis. Triiodothyronine (T3), tetraiodothyronine (T4) and thyroid stimulating hormone (TSH) levels are the commonly employed lab studies used to assess the level of function of the thyroid gland. Among these parameters, TSH has been reported to be the most sensitive marker of hypothyroidism. Even after the diagnosis is established, frequent monitoring of TSH is done to assess the response to treatment and progression of the disease. After the assessment of patient's thyroid function status, the focus shifts to indentifying the presence of antithyroid antibodies. It should be noted that while the presence of antithyroid peroxidase (ATPO) and antithyroglobulin (TGAB) antibodies are both positively correlated with Hashiomoto's thyroiditis, the correlation is slightly higher for TGAB than ATPO. (Kasagi et al., 1996). Even in the absence of hypothyroid symptoms, the presence of antithyroid antibodies would indicate underlying lymphocytic infiltration of the gland, and be indicative of autoimmune disease (Yoshida et al., 1978). In an attempt increase the certainty of the diagnosis, antimicrosomal antibodies have been found to afford greater diagnostic accuracy when compared to antithyroglobulin antibodies. However, for those cases in which Hashimoto's thyroiditis is suspected clinically but antibody titers are not elevated, fine needle aspiration (FNA) and cytological examination continue to play a defining role in establishing the diagnosis (Baker et al., 1982; Kumar et al., 2002; Takashi et al., 2008).

In delineating key cytological findings, extent of lymphocytic infiltration and the presence of Hurthle cells has been found to be directly proportional to the severity of the disease. Also, as the disease progresses, colloid in the thyroid gland is destroyed and the spaces between follicular cells shrink, altering the microscopic appearance of FNA biopsy specimens. In further elaboration on how microscopic appearance correlates with disease severity, extent of involved tissue has been found to be directly proportional to severity of the disease. Despite the diagnostic sensitivity and accuracy of cytological analysis, in some instances, the 
presence of numerous hyperplastic follicular cells may lead to a false diagnosis of follicular carcinoma. Alternatively, the diagnosis of some neoplasms, like Hurthle cell tumor, could be misdiagnosed as Hashimoto's thyroiditis due to the presence of a large number of Hurthle cells (MacDonald \& Yazdi, 1999). In addition to the above investigations, accurate diagnosis must also incorporate clinical correlation.

Radioactive iodine uptake (RAIU) is another modality which is commonly employed in diagnosing thyroid disorders. The role of RAIU in the diagnosis of Hashimoto's thyroiditis has been debated for many years (Cohen et al., 1965). A potentially less obtrusive study which may be performed to discern thyroid pathology is an ultrasound. Ultrasonography provides information regarding anatomic characteristics of the gland and identifies any major changes in the gland. Ultrasonography can be helpful in discerning Hashimoto's thyroiditis in goiters of unknown etiology and can identify the cause of functional impairment as well as the necessity for treatment (Sostre \& Reyes, 1991).

The physical characteristics of the thyroid gland, serum TSH levels, serum antithyroid antiglobulin titer, radioactive iodine uptake of the gland, and the response to the perchlorate discharge test are widely used in making an accurate diagnosis of the disease. Indeed, the clinician can feel reasonably confident in their diagnosis of Hashimoto's thyroiditis if at least two of the above mentioned tests support the diagnosis (Fisher et al., 1975). Some recent studies have subclassified Hashimoto's thyroiditis as IgG-4 thyroiditis and nonIgG-4 thyroiditis. This distinction may be important in that IgG-4 thyroiditis has been associated with severe lymphoplasmacytic infiltration, marked fibrosis, and lymphoid follicle formation in contrast to nonIgG-4 thyroiditis, which exhibits more mild histopathological changes (Li., 2009). Thus, this classification might be helpful in assessing the severity of the disease and could be used in determining the most appropriate treatment options for patients.

Furthermore the disease process must be differentiated from some commonly occurring thyroid disorders such as nontoxic nodular goiter and Graves' disease. The presence of a multinodular goiter with gross nodularity is usually considered to be evidence against the diagnosis of Hashimoto's thyroiditis but it cannot be ruled out based on this finding (Takashi et al., 2008). Unlike Hashimoto's thyroiditis, multinodular goiter is usually characterized by euthyroid status and absence of antithyroid antibodies. Hashimoto's thyroiditis and multinodular goiter commonly coexist in patients thus, FNA is commonly employed to differentiate these two entities. Tumor of thyroid gland is another entity which has to be differentiated from Hashimotos' thyroiditis. Rapid growth of the gland and persistent pain usually arouses suspicion of tumor. The confirmatory diagnosis of tumor is usually performed with the aid of FNA. Thyroid lymphoma may develop in some cases of Hashimoto's thyroiditis. Some studies have indicated that using reverse transcriptase polymerase chain reaction might be helpful in differentiating thyroid lymphoma and Hashimoto's thyroiditis (Takano et al., 2000). Furthermore, although Hashimoto's thyroiditis typically presents with hypothyroid symptoms, patients may occasionally present with hyperthyroidism and thyrotoxicosis. This necessitates the differentiation of Hashimoto's thyroiditis from Graves' disease, in cases associated with symptoms of excess thyroid hormone. 


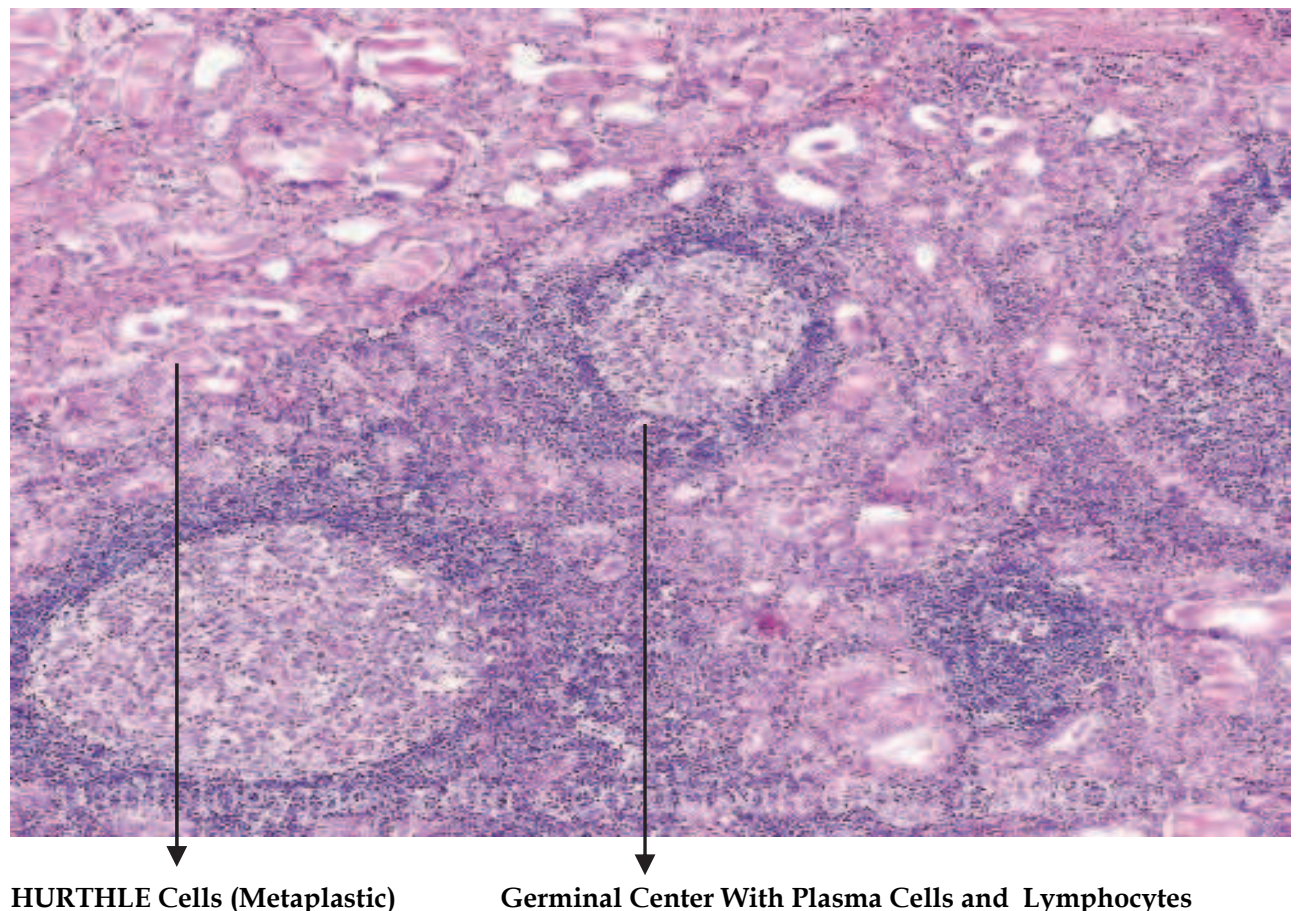

Fig. 3. Histological section thyroid gland affected with Hashimoto's Disease (Datto \& Youens, 2007).

\section{Treatment}

Options in the treatment of Hashimoto's thyroiditis include medical therapy and surgical resection of the gland. The appropriate choice depends on disease presentation and extent of gland involvement. In some instances, patients may present without symptoms, and may not require immediate intervention. (Vickery \& Hamlin, 1961). However, continuing debate surrounds whether prophylactic replacement of thyroid hormone has therapeutic benefit in euthyroid-appearing patients with Hashimoto's thyroiditis (Chiovato et al., 1986). Recently, studies have shown that prophylactic treatment in euthyroid patients can slow the progression of the disease and significantly reduce levels of antithyroid antibodies; however, the long-term benefits of this approach have not yet been confirmed (Padberg etal 2004). Furthermore, ultrasound studies have shown that thyroid size diminishes in response to thyroid hormone replacement, even in euthryoid patients (Hegedus et al., 1991). Moreover, reversibility in the progression of the disease appears to be quicker and more pronounced in younger patients than in more mature patients. This difference may be attributable to the extent of glandular involvement and increased degree of fibrosis in older populations, making reversibility of the underlying pathology less feasible. 


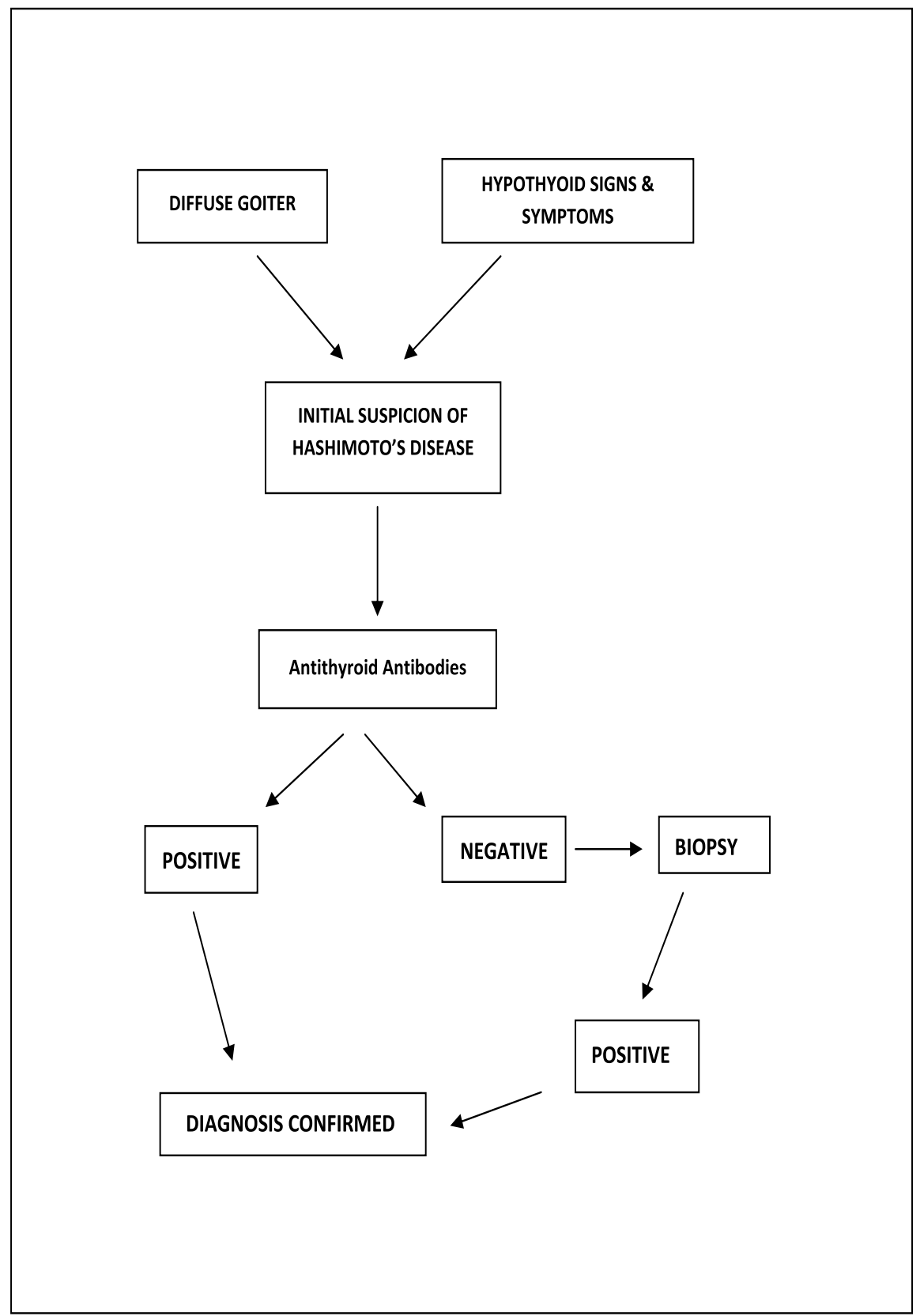

Fig. 4. Flow diagram representing the diagnosis of Hashimoto's thyroiditis 
After assessment of the functional status of thyroid gland, thyroid hormone replacement therapy is instituted in all Hashimoto's thyroiditis patients with documented hypothyroidism. Thyroid hormone replacement is also indicated in the presence of a goiter, if the goiter is small in size and is causing minimal pressure symptoms or disfigurement. The initial dosage of the thyroid hormone is determined based upon the patient's body mass, cardiovascular condition, concomitant co-morbid conditions and pregnancy status. The daily dosing in healthy young individuals is usually calculated as 1.7 micrograms/Kilogram of body weight per day which typically ranges between 75-125 micrograms per day. Most hypothyroid patients suffering with Hashimoto's thyroiditis will need lifelong replacement of thyroid hormone. External supplementation of thyroid hormone will not only correct the metabolic status of the person but it is also postulated to modify the course of the disease. Long term follow up of patients treated with thyroxine has shown reduced antithyroid peroxidase antibodies after a mean time of 50 months, with a small number of patients being reported as seronegative (Schmidt et al., 2008). Moreover, about $20 \%$ of patients suffering from Hashimoto's thyroiditis-related hypothyroidism recovered normal thyroid function when challenged with thyroid releasing hormone (TRH). In addition, a few studies have shown that if patients recover normal thyroid gland function, they might remain euthyroid, despite not taking hormone therapy, for a mean period of approximately 8 years (Takasu et al., 1990).

In contrast to hormone replacement therapy, nutritional therapies, which focus on modifying the body's immune response and resultant destruction of thyroid tissue, continue to be an area of keen interest. Selenium, a trace element which plays an important role in modifying inflammatory and immune responses in the body, has been proposed to have disease-modifying properties in Hashimoto's thyroiditis. The rationale for using this nutrient stems from the discovery that the enzymes iodothyronine deiodinase, glutathione peroxidase and thioredoxin reductase, which maintain thyroid gland homeostasis, are selenium dependant. One study investigating the effect of selenium supplementation on Hashimoto's thyroiditis found significant reduction in the levels of antithyroid peroxidase (ATPO) following 6 months of therapy. Further decline in antibody levels was observed when the therapy was continued, with antibody levels increasing after therapy was terminated. (Mazokapakis et al., 2007).

In some cases, pharmacotherapy and hormone replacement might not be sufficient to treat the symptoms of Hashimoto's thyroiditis and surgical therapy is required. Surgical therapy is indicated in patients suffering from severe, painful goiter or experiencing pressure symptoms resulting from tracheal encroachment, which include dysphasia or dyspnea. In an attempt to create guidelines for thyroid resection, the following factors have been found to play a major role in determining when to pursue surgical resection (Thomas \& Rutledge, 1981):

1. Dominant mass unresponsive to thyroxine therapy

2. Increase in the size of the mass despite thyroxine therapy

3. History or physical examination findings suggestive of malignancy

4. Indeterminate findings on cutting needle biopsy

A small group of patients, with Hashimoto's thyroiditis, present with pain and tenderness rather than a goiter or hypothyroidism. Thyroidectomy has been proven to be effective in 
these patients as treatment with thyroid hormone replacement or corticosteroids will not alleviate their symptoms (Kon \& Degroot, 2003). Painful Hashimoto's thyroiditis is an atypical variant characterized by recurrent attacks of fever and thyroid pain in the presence of antithyroid antibodies. These cases do not respond to the regular antiinflammatory agents, which have been found to be effective in controlling pain associated with other forms of thyroiditis. In assessing the risk/benefit trade-off of thyroid resection, the complication risk involved in performing thyroidectomy in patients with Hashimoto's thyroiditis is reported to be very low, but the presence of unsuspected coexisting malignancies is common (Shih et al., 2008). Moreover, prophylactic removal of a nodular thyroid gland is done in selected cases to prevent the development of thyroid cancer, which would be typically diagnosed at a later stage. It should be noted that the effectiveness of this approach has been widely debated and remains a point of research interest. In cases with documented thyroid cancer, removal of the gland followed by radiotherapy or chemotherapy, depending on the type of tumor, is the definitive therapy. The presence of tumors coexistent with Hashimoto's thyroiditis does not alter surgical management when compared to cases of Hashimoto's thyroiditis uncomplicated by neoplasm (Singh et al., 1999). Surgical removal of the thyroid gland has been tried with variable success in cases of Hashimoto's thyroiditis associated with chronic urticaria, when anti-allergic and corticosteroid therapies have proven ineffective. Briefly summarized; thyroid hormone status, pressure symptoms associated with an enlarged gland, and presence of associated symptoms or other autoimmune disorders should be considered in making an accurate treatment choice.

\section{Hashimoto's encephalopathy}

Hashimoto's encephalopathy or encephalitis is a rare neuroendocrine entity and is described as an autoimmune encephalopathy, which occurs in patients diagnosed with Hashimoto's thyroiditis. Similar to Hashimoto's thyroiditis, it can affect individuals of all age groups, and is more common in women than in men. Hashimoto's encephalopathy is frequently misdiagnosed since symptoms at presentation are predominantly neurological. Some cases have been reported where patients presented with Hashimoto's encephalopathy long before there was any clinical suspicion for Hashimoto's thyroiditis (Peschen-Rosin et al., 1999). Hashimoto's encephalopathy was first described in 1961, in a 48 year-old man who was hypothyroid and who experienced recurring episodes of encephalopathy and stroke-like symptoms (Brain et al., 1966). Some authors prefer using the term corticosteroid-responsive encephalopathy rather than Hashimoto's encephalopathy as the pathogenesis of this condition is still a topic of widespread conjecture (Fatourechi, 2005). The estimated prevalence of this condition is $2.1 / 100,000$ (Ferracci \& Giani, 2003). The actual prevalence of the disease could be much higher since many cases of Hashimoto's encephalopathy are presumed to remain undiagnosed. Studies attempting to describe the pathophysiological mechanisms behind this condition have suggested the possible role of autoimmune processes. Similar to Hashimoto's thyroiditis, patients with Hashimoto's encephalopathy have high levels of antithyroid antibodies and respond to immunosuppressive therapy, supporting the involvement of an autoimmune mechanism in its pathogenesis (Schiess \& Pardo, 2008). An underlying immune 
mechanism is further supported by autopsy studies which revealed histopathological changes such as lymphocyte infiltration of the leptomeninges, and gliosis of cortical gray matter, basal ganglia, thalamus and hippocampus, which are reminiscent of autoimmune injury to other organs of the body (Duffey \& Yee, 2003). The presentation of Hashimoto's encephalopathy can be either acute or subacute, and is characterized by a relapsingremitting or progressive course of seizures, tremors, ataxia, myoclonus, psychosis, and stroke-like neurological findings. The literature indicates that the initial clinical presentation can be classified either as a vasculitic type, with predominantly stroke-like symptoms and mild cognitive impairment, or a diffuse progressive type with predominant cognitive impairment (Kothbauer-Marggreiter et al., 1996). In contrast to disease prevalence findings in adults, very few instances of Hashimoto's encephalopathy have been reported in the pediatric age group. Pediatric Hashimoto's encephalopathy is characterized by seizures, hallucinations, and confusion, and suspicion should arise when a progressive decline in school performance is observed (Vasconcellos et al., 1998).

The diagnosis of Hashimoto's encephalopathy continues to be a diagnosis of exclusion. Serum titers of antithyroid antibodies will be elevated and cerebrospinal fluid analysis will show increased protein levels. Other possible causes of encephalopathy including infections, metabolic and electrolyte derangements, toxic ingestions, vascular abnormalities, and neoplastic or paraneoplastic syndromes must be ruled out before a Hashimoto's encephalopathy diagnosis is made. Electroencephalogram and imaging studies in patients with suspected Hashimoto's encephalopathy typically exhibit nonspecific changes, in the absence of infection, tumor, or stroke (Marshall \& Doyle, 2006). The antibody titers in Hashimoto's encephalopathy are not suggestive of the severity or the type of clinical presentation. Early diagnosis and prompt intervention are of critical importance in effectively treating this condition, and significantly reducing its morbidity and mortality. The first line of treatment is usually corticosteroids, and in cases where steroids are contraindicated, other immunosuppressive agents have been employed with good efficacy. In steroid unresponsive cases, administration of plasmapheresis has been shown effective in controlling symptoms (Nagpal \& Pande, 2004). Periodic intravenous exchange may also be used for steroid non-responders, but no superiority has been established when compared to plasmapheresis. The duration of the treatment is highly variable, however approximately $90 \%$ of the cases will remain in remission after treatment.

\section{Conclusion}

In this chapter, we have discussed the epidemiology, presumed pathogenesis, diagnosis and treatment of Hashimoto's thyroiditis. We have also discussed potential complications including other autoimmune diseases and neoplasms. In many ways, Hashimoto's thyroiditis serves as a paradigm for autoimmune disease throughout the body. Our understanding of how a genetic predisposition can be modified by environmental exposure is expanding. Our grasp of how aggressive immune suppression can alter disease course is growing. As with many subjects in medicine, with knowledge comes more questioning of what we know. Just as in other disease states, we must eagerly seek out both the questions and the answers. 


\section{Acknowledgements}

The authors acknowledge Helen Houpt, MSLS for her editorial assistance in the production of this manuscript.

\section{References}

Baker, B, A., Gharib, H., Markowitz, H. (1983). Correlation of Thyroid Antibodies and Cytologic Features in Suspected Autoimmune Thyroid Disease. The American Journal of Medicine, Vol. 74, No. 6, (June, 1983), pp. (941-944), doi: 10.1016/00029343(83)90786-6.

Becker, K, L., Ferguson, R, H., Mc cohaney, W, M. (1963). The Connective Tissue Diseases and Symptoms Associated with Hashimoto's Thyroiditis. The New England Journal of Medicine, Vol. 263, (February, 1963), pp. (277-280).

Boukis, M, A., Koutras, D, A., Souvatzoglou, A., Evangepolau, K., Vrontakis, M., Moulapoulaos, S, D. (1983). Thyroid Hormone and Immunologic Studies in Endemic Goiter. The Journal of Clinical and Endocrinology and Metabolism, Vol, 57, (1983), pp. (859-862).

Brain, L., Jellinek, E, H., Ball, K. (1966). Hashimoto's disease and encephalopathy. Lancet, Vol. 2, (1966), pp (512-514).

Buchanan, W, W., Harden, R, M. (1965). Primary Hypothyroidism and Hashimoto's Thyroiditis. Archives of Internal Medicine, Vol. 115, No. 4, (April, 1965), pp. (411417).

Casselman, W, G. (1996). Thyroid, In: Index of Medical Word origins, (1996), Available from: <Hashimoto's Thyroiditistp://www.billcasselman.com/dictionary_of_medical_ der ivations/dmd_nine.Hashimoto's Thyroiditism>.

Champion, B, R., Page, K, R., Parish, N., Rayner, D, C., Dawe, K., Biswas-Hughes, G., Cooke, A., Geysen, M., Roitt, I, M. (1991). Identification of a Thyroxine-Containing SelfEpitope of Thyroglobulin Which Triggers Thyroid Auto reactive T Cells. The Journal of Experimental Medicine, Vol. 174, (August, 1991), pp. (363-370), ISSN 00221007/91/08/0363/08.

Chistiakov, D.A., Turakulov, R.I. (2003). CTLA4 and its role in autoimmunr thyroid disease. Journal of Molecular Endocrinology, Vol. 31, (August, 2003), pp. (21-36), doi: 10.1677 /jme.0.0310021.

Chiovato, L., Marcocci, C., Mariotti, S., Mori, A., Pinchera, A. (1986). L-thyroxine therapy induces a fall of thyroid microsomal and thyroglobulin antibodies in idiopathic myxedema and in hypothyroid, but not in euthyroid Hashimoto's thyroiditis. Journal of Endocrinological Investigation, Vol. 9, No. 4, (August, 1986), pp. (299-305).

Cohen R,J., Stansifer P,D., Barrett, O. (1965). Radioactive Iodine Uptake In Hashimoto's Thyroiditis. Archives of Internal Medicine, Vol. 116, (July, 1965), pp. (111-112), PubMed PMID: 14338941.

Cuoco, L., Certo, M., Jorizzo, R, A., De Vitis, I., Tursi,, A., Papa, A., De Marinis, L., Fedeli, P., Fedeli, G., Gasbarrini, G. (1999). Prevalence and Early Diagnosis of Celiac Disease in Autoimmune Thyroid Disorders. Italian Journal of Gastroenterology and Hepatology, Vol. 31, No. 4, (May, 1999), pp. (283-287). 
Datto, M., Youens, K. (2007). Hashimotos Thyroiditis, In: Pathology Pics, February, 2008, Available from <www.pathologypics.com/pictview.aspx?id=291>.

Dietrich, G., Piechaszuk, M., Pau, B., Kassatchkine, M, D. (1991). Evidence for a Restricted Idiotypic and Epitope Specificity of Anti-thyroglobulin Auto antibodies in Patients with Autoimmune Thyroiditis. European Journal of Immunology, Vol. 21, No. 3, (March, 1991), pp. (811-814), doi: 10.1002/eji.1830210340.

MacDonald, L., Yazdi, H, M. (1999). Fine Needle Aspiration Cytology of Hashimoto's Thyroiditis: Sources of Diagnostic Error. Acta Cytologica, Vol. 43, No. 3, (June, 1999), pp. (400-406).

Dufey, P., Yee, S., Reid, I, N., Bridges, L, R. (2003). Hashimoto's Encephalopathy: Postmortem Findings after Fatal Status Epilepticus. Neurology, Vol. 61, No. 8, (October, 2003), pp. (1124-1126), doi: 10.1212/01.WNL.0000090462.62087.

Einarsdottir, E., Soderstrom, I., Lofgren-Burstrom, A., Haraldsson, S., Nilsson-Ardnor, S., Penha-Goncalves, C., Lind, L., Holmgren, G., Holmberg, M., Asplund, K., Holmberg, D. (2003). The CTLA-4 Region as a General Autoimmunity Factor: An Extended Pedigree Provides Evidence for Synergy with the HLA locus in the Etiology of Type 1 Diabetes Mellitus, Hashimoto's Thyroiditis and Grave's Disease. European Journal of Human Genetics, Vol. 11, (2003), pp. (81-84).

Fatourechi, V. (2005). Hashimoto's Encephalopathy: Myth or Reality? An Endocrinologists Perspective. Best Practice and Research Clinical Endocrinology and Metabolism, Vol. 9, No. 1, (2005), pp. (53-66), doi: 10.1016/j.beem.2004.11.006.

Fisher, D.A., Oddie, T.H., Johnson, D.E., Nelson, J.C (1975). The Diagnosis Of Hashimoto's Thyroiditis. The Journal of Clinical Endocrinology and Metabolism, Vol. 40, No.5, (May, 1975), pp. (795-801), doi:10.1210/jcem-40-5-795.

Fischer, G, F. (2000). Molecular Genetics of HLA. Vox Sanguinis, Vol. 78, No. 10, (2000), pp. (261-264), ISSN 0042-9007.

Ferracci, F., Bertiato, G., Moretto, G. (2004). Hashimoto's encephalopathy Epidemiological Data and Pathogenetic Considerations. Journal of the Neurological Sciences, Vol. 217, No. 2, (February, 2004), pp. (165-168). PubMed PMID:14706219

Fukata, s., Kuma, K., Sugawara, M. (1996). Relationship Between Cigarette Smoking and Hypothyroidism in Patients with Hashimoto's Thyroiditis. Journal of Endocrinological Investigation, Vol. 19, No. 9, (1996), pp. (607-612), ISSN 0391-4097.

Gebe, J, A., Swanson, E., Kwok, W, W. (2002). HLA Class II Peptide-Binding and Autoimmunity. Tissue Antigens, Vol. 59, No. 2, (February, 2002), pp. (78-87).

Giordano, C., Richiusa, P., Bagnasco, M., Pizzolanti, G., Di Blasi, F., Sbriglia, M, S., Mattina, A., Pesce, G., Montagna, P., Capone, F., Misiano, G., Scorsone, A., Pugilese, A., Galluzzo, A. (2001). Differential Regulation of Fas-Mediated Apoptosis in Both Thyrocyte and Lymphocyte Cellular Compartments Correlates with Opposite Phenocytic Manifestations of Autoimmune Thyroid Disease. Thyroid, Vol. 11, No. 3, (March, 2001), pp. (233-244), doi: 10.1089/105072501750159615.

Gordin, A., Saarinen, P., Pelkonen, A., Lamberg, B. (1974). Serum Thyroglobulin and the Response to Thyrotropin Releasing Hormone in Symptomless Autoimmune 
Thyroiditis and in Borderline and Overt Hypothyroidism. Acta Endocrinologica, Vol. 75, No. 274, (1974).

Hall, R, C., Popkin, M, K., DeVaul, R., Hall, A, K., Gardner, E, R., Beresford, T, P. (1982). Psychiatric Manifestations of Hashimoto's Thyroiditis, Psychosomatics, Vol. 23, No. 4, (April, 1982), pp. (337-342).

Hegedus, L., Hansen, J, M., Rasmussen, U, F., Hansen, B, M., Mased, M, H. (1991). Influence of Thyroxine Treatment on Thyroid Size and Anti-Thyroid Peroxidase Antibodies in Hashimoto's Thyroiditis. Clinical Endocrinology, Vol. 35, No. 9, (September, 1991), pp. (235-238), doi: 10.1111/j.1365-2265.1991.tbo3528.x.

Hollowell, J, G., Staehling, N, W., Flanders, W, D., Hannon, W, H., Gunter, E, W., Spencer, C, A., Braverman, L, E. (2002). Serum TSH, $\mathrm{T}_{4}$, and Thyroid Antibodies in the United States Population (1988 to 1994): National health and Nutrition Examination Survey (NHALES III). The Journal of Endocrinology and Metabolism, Vol. 87, (2002), pp. (489-499), doi: 10.1210/jc.87.2.489.

Hunt, P, J., Marshall, S, E., Weetman, A, P, Bunce, M, Bell, J, I., Wass, J, A., Welash, K, L. (2001). Histocompatability Leukocyte Antigens and Closely Linked Immunomodulatory Genes in Autoimmune Thyroid Disease. Clinical Endocrinology, Vol. 55, No. 4, (October, 2001), pp. (491-499), doi: 10.1046/j.1365-2265.2001.01356.x.

Hygek, E., Isaacson, P, G. (1988). Primary B-cell Lymphoma of Thyroid and its Relationship to Hashimoto's Thyroiditis. Human Pathology, Vol. 9, No. 11, (November, 1988), pp. (1315-1326), doi: 10.1016/s0046-8177(88)80287-9.

Kakourou, T., Kanaka-Gantenbein, C., Papadopoulou, A., Kaloumenou, E., Chrousos, G, P. (2005). Increased Prevelance of Chronic Autoimmune (Hashimoto's) Thyroiditis in Children and Adolescents Suffering from Vitiligo. Journal of American Academy of Dermatology, Vol. 53, No. 2, (August, 2005), pp. (220-223), doi: 10.1016/j.jaad.2005.03.032.

Kasagi, K., Kousaka, T., Higuchi, K., Ida, Y., Misaki, T., Miyamoto, S., Alam, M.S., Yamabe, H., Konishi, J. (1996). Clinical Significance of Measurements of Antithyroid Antibodies in the Diagnosis of Hashimoto's Thyroiditis: Comparison with Histological Findings. Thyroid, Vol. 6, No.5, (October, 1996), pp. (445-450), doi: 10.1089/thy.1966.6.445.

Kon, Y,C., Degroot, L,G. (2003). Painful Hashimoto's Thyroiditis as an indication for Thyroidectomy: Clinical Characteristics and Outcome in Seven Patients. The Journal of Clinical Endocrinology and Metabolism, Vol. 88, No.6, (June, 2003), pp. (2667-2672), doi: $10.1210 /$ jc.2002-021498.

Kothbauer-Margreiter, I., Sturzenegger, M., Komor, J., Baumgartner, R., Hess, C, W. (1996). Encephalopathy Associated with Hashimoto's Thyroiditis: Diagnosis and Treatment. Journal of Neurology, Vol. 243, No. 8, (April, 1996), pp. (585-593), doi: 10.1007/BF00900946.

Kumar, N., Ray, C., Jain, s. (2002). Aspiration Cytology of Hashimoto's Thyroiditis in a Endemic Area. Cytopathology, Vol. 13, No. 1, (February, 2002), pp. (31-39), doi: 10.1046/j.1365-2303.2002.00366.x. 
Limachi, F., Basso, S. (2002). Apoptosis: Life Though Planned Cellular Death Regulating Mechanisms, Control Systems and Relations with Thyroid Disease. Thyroid, Vol. 12, No. 1, (January, 2002), pp. (27-34), doi: 10.1089/105072502753451931.

Lima, M, A., Santos, B, M., Borges, M, F. (1998). Quantitative Analysis of C Cells in Hashimoto's Thyroiditis. Thyroid, Vol. 8, No. 6, (June, 1998), pp. (505-509), doi: 10.1089/thy.1998.8.505.

Li, Y., Bai, Y., Liu, Z., Ozaki, T., Taniguchi, E., Mori, I., Nagayama, K., Nakamura, H., Kakudo, K. (2009). Immunohistochemistry of IgG-4 can Help Sub classify Hashimoto's Autoimmune Thyroiditis. Pathology International, Vol. 59, No.9, (September, 2009), pp. (636-641), doi: 10.1111/j.1440-1827.2009.02419.x.

Marshall, G, A., Doyle, J, J. (2006). Long-term Treatment of Hashimoto's Encephalopathy. Journal of Neuropsychiatric and Clinical Neurosciences, Vol. 18, No. 1, (2006), pp. (1420).

Martin,A., Davies, T, F. (1992). T Cells and Human Autoimmune Thyroid Disease: Emerging Data Show Lack of Need to Invoke Suppressor T Cell Problems. Thyroid, Vol. 2, No. 3, (1992), pp. (247-261), doi: 10.1089/thy.1992.2.247.

Male, D, K., Champion, B, R., Pryce, G., Matthews, H., Sheperd, P. (1985). Antigenic Determinants of Human Thyroglobulin Differentiated Using Antigen Fragments. Journal of Immunology, Vol. 54, No. 3, (March, 1985), pp. (419-427).

Mazokopakis, E, E., Papadakis, J, A., Papadomanolaki, M, G., Batistakis, A, G., Giannakopoulos, T, G., Protopapadakis, E, E., Ganotakis, E, S. (2007). Effects of 12 Months Treatment with I-Selenomethionine on Serum Anti-TPO Levels in Patients with Hashimoto's Thyroiditis. Thyroid, Vol. 17, No. 7, (August, 2007), pp. (609-612), doi: 10.1089 /thy.2007.0040.

Mccohaney, W, M., Keating, F, R., Beahrs, O, H., Woolner, L, B. (1962). On the Increasing Occurrence of Hashimoto's Thyroiditis. The Journal of Clinical Endocrinology and Metabolism, Vol. 22, No. 542, (1962), doi: 10.1210/jcem-22-5-542.

Nagpal, T., Pande, s. (2004). Hashimoto's Encephalopathy: Response to Plasma Exchange. Neurology India, Vol. 52, (2004), pp. (245-247).

Nikiforova, M, N., Caudill, C, M., Biddinger, P., Nikiforov, Y, E. (2002). Prevalence of RET/PTC Rearrangements in Hashimoto's Thyroiditis and Papillary Thyroid Carcinomas. International Journal of Surgical Pathology, Vol. 10, No. 1, (January, 2002), pp. (15-22), doi: 10.1177/106689690201000104.

Padberg, S., Heller, K., Usadel, K, H, Schumm-Draaeger, P, M. (2001). One Year Prophylactic Treatment of Euthyroid Hashimoto's Thyroiditis Patients with Levothyroxine : Is there a Benefit. Thyroid, Vol. 11, No. 3, (March, 2001), pp. (249-255), doi: $10.1089 / 105072501750159651$.

Peschen-Rosin, R., Schabet, M., Dichgans, J. (1999). Manifestation of Hashimoto's Encephalopathy Years Before Onset of Thyroid Disease. European Neurology, Vol. 41, No. 2, (1999), pp. (79-84), doi: 10.1159/000008007.

Pino Rivero, V., Guerra Camacho, M., Marcos García, M., Trinidad Ruiz, G., Pardo Romero, G., González Palomino, A., Blasco Huelva, A. (2004). The Incidence of Thyroid Carcinoma in Hashimoto's Thyroiditis: Our Experience and Literature 
Review. An Otorrinolaringol Ibero Am, Vol. 31, No. 3, (2004), pp. (223-230). Review. Spanish

Pyne, D., Isenberg, D, A. (2002). Autoimmune Thyroid Disease in Systemic Lupus Erythematosus. Annals of Rheumatology, Vol. 61, (2002), pp. (70-102), doi: 10.1136/ard.61.1.70.

Rottem, M. (2003). Chronic Urticaria and Autoimmune Thyroid Disease: Is There a Link? Autoimmune Reviews, Vol. 2, No. 2, (March, 2003), pp. (69-72), doi: 10.1016/s15689972(02)00141-6.

Radetti, G., Gottardi, E., Bona, G., Corrias, A., Salardi, S., Loche, S. (2006). The Natural History of Euthyroid Hashimoto's Thyroiditis in Children. Journal of Pediatrics, Vol. 149, No. 6, (December, 2006), pp. (827-832), doi: 10.1016/j.peds.2006.08.045.

Shih, M, L., Lee, J, A., Hsieh, C, B., Liu, H, D., Kebebew, E., Clark, O, H., Duh, Q, Y. (2006). Thyroidectomy for Hashimoto's Thyroiditis: Complications and Associated Cancers. Thyroid, Vol. 18, No. 7, (July, 2006), pp. (729-734), doi: 10.1089 / thy.2007.0384.

Staii, A., Mirocha, S., Todorova-Koteva, K., Glinberg, S., Jaume, J, C. (2010).Hashimoto's Thyroiditis is More Frequent than Expected when Diagnosed by Cytology which Uncovers a Pre-Clinical State. Thyroid Research Journal, Vol. 3, No.11, (2010).

Sostre, S., Reyes, M, M. (1991). Sonographic Grading and Diagnosis of Hashimoto's Thyroiditis. Journal of Endocrinological Investigation, Vol. 14, No. 2, (February, 1991), pp. (115-121).

Schiess, N., Pardo, C, A. (2008). Hashimoto's Encephalopathy. Annals of New York Academy of Sciences, Vol. 1142, , (October, 2008), pp. (254-265), doi: 10.1196/annals.1444.018.

Schmidt, M., Voell, M., Rahlff, I., Dietlein, M., Kobe, C., Faust, M., Schicha, H. (2008). Long-Term Follow-Up of Antithyroid Peroxidase Antibodies in Patients with Chronic Autoimmune Thyroiditis (Hashimoto's Thyroiditis) Treated with Levothyroxine. Thyroid, Vol. 18, No. 7, (2008), pp. (755-760), doi: 10.1089/thy.2008.0008.

Singh, B., Shaha, A, R., Trivedi, H., Carew, J, F., Poluri, A., Shah, J, P. (1999). Coexistent Hashimoto's Thyroiditis with Papillary Thyroid Carcinoma: Impact on Presentation, Management, and Outcome. Surgery, Vol. 126, No. 6, (December, 1999), pp. (1070-1077), doi: 10.1067/msy.2099.101431.

Tagami, T., Tamanaha, T., Shimazu, S., Honda, K., Nanba, K., Nomura, H., Yoriko, S, U., Usui, T., Shimatsu, A., Naruse, M. (2010). Lipid Profiles in Untreated Patients with Hashimoto's Thyroiditis and the Effects of Thyroxine Treatment on Subclinical Hypothyroidism with Hashimoto's Thyroiditis. Endocrine Journal, Vol. 57, No. 3, (December, 2009), pp. (253-258).

Takano, T., Miyauchi, A., Matsuzuka, F., Yoshida, H., Kumar, K., Amino, N. (2000). Diagnosis of Thyroid Malignant Lymphoma by Reverse Transcription- Polymerase Chain Reaction Detecting the Monoclonality of Immunoglobulin Heavy Chain Messenger Ribonucleic Acid. Journal of Clinical Endocrinology and Metabolism, Vol. 85, (2000), pp. (67-675), doi: 10.1210/jc.85.2.671.

Takasu, N., Komiya, I., Asawa, T., Nagasawa, Y., Yamada, T. (1990). Test for Recovery from Hypothyroidism during Thyroxine Therapy in Hashimoto's Thyroiditis. Lancet, 
Vol. 336, No. 8723, (November, 1990), pp. (1084-1086), doi:10.1016/01406736(90)92567-2.

Takasu, N., Yamada, T., Takasu, M., Komiya, I., Nagasawa, Y., Asawa, T, Shinoda, T., Aizawa, T., Koizumi, Y. (1992). Disappearance of Thyrotropin-Blocking Antibodies and Spontaneous Recovery from Hypothyroidism in Autoimmune Thyroiditis. The New England Journal of Medicine, Vol. 326, (February, 1992), pp. (513-518), doi: 10.1056/NEJM199202203260803.

Takashi, A., Nobuyuki, A., De Groot, L.J. (2008). Hashimoto's Thyroiditis, In: Thyroid Disease Manager, Takashi, A., Nobuyuki, A, Endocrine Education, Inc., Retrieved from < Hashimoto's Thyroiditistp://www.thyroidmanager.org/Chapter8/chapter8.Hashimoto's Thyroiditisml\#id2504737>.

Tunbridge, W, M., Evered, D, C., Hall, R., Appleton, D., Brewis, M., Clark, F., Evans, J, G., Young, E., Bird, T, Smith, P, A. (1977). The Spectrum of Thyroid Disease in a Community: The Wickham Survey. Clinical Endocrinology, Vol. 7, No. 6, (December, 1977), pp. (481-493).

Thomas, C, G., Rutledge, R, G. (1981). Surgical Intervention in Chronic (Hashimoto's) Thyroiditis. Annals of Surgery, Vol.193, No. 6, (June, 1981), pp. (769-776).

Unger, P., Ewart, M., Wang, B, Y., Gan, L., Koz, s., Burstein, D, E. (2003). Expression of P63 in Papillary Thyroid Carcinoma and in Hashimoto's Thyroiditis: A Pathological Link? Human Pathology, Vol. 34, NO. 8, (August, 2003), pp. (764-769), doi: 10.1016/s0046-8177(03)00239-9.

Vasconcellos, E., Pina-Girza, J. E., Fakhoury, T., Fenichel, G.M. (1999). Pediatric Manifestations of Hashimoto's Encephalopathy. Pediatric Neurology, Vol. 20, No.5, (May, 1999), pp. (394-398), doi: 10.1016/s0887-8994(99)00006-5.

Vickery, A, L., Hamlin, E (1961). Struma Lymphomatosa (Hashimoto's Thyroiditis): Observations on Repeated Biopsies in 16 Patients. The New England Journal of Medicine, Vol. 264 , (February, 1961), pp. (226-229).

Volpe, R., Clark, P, V., Row, V, V. (1973). Relationship of Age Specific Incidence Rates to Immunological Aspects of Hashimoto's Thyroiditis. Canadian Medical Association Journal, Vol. 109, No. 9, (November, 1973), pp. (898-901).

Wang, C., Crapo, L, M. (1997). The Epidemiology of Thyroid Disease and Implications for Screening. Endocrinology and Metabolism Clinics of North America, Vol. 26, No.1, (1997), pp. (189-218), ISSN 0889-852.

Wu, Z., Stephens, H, A., Sachs, J, A., Biro, P, A., Cutbush, S., Magzoub, M, M, Becker, C., Schwartz, G., Bottazzo, G, F. (1994). Molecular Analysis of HLA-DQ and DP Genes in Caucasoid Patients with Hashimoto's Thyroiditis. Tissue Antigens, Vol. 43, No. 2, (February, 1994), pp. (116-119).

Weetman, A, P., Walport, M, J. (1987). The Association of Autoimmune Thyroiditis with Systemic Lupus Erythematosus. Oxford Journals Rheumatology, Vol. 6, No. 5, (19897), pp (359-361), doi: 10.1093/rheumatology/26.5.359

Wilkinson, M., Sacker, L, S. (1957). The Lupus Erythematosus Cell and its significance. British Medical Journal, Vol. 2, No. 5046, (September, 1957), pp. (661-665).

Yoshida, H., Amino, N., Yagawa, K., Uemura, K., Satoh, M., Miyai, K., Kumahara, Y. (1978). Association of Serum Antithyroid Antibodies with Lymphocytic Infiltration of the 
Thyroid Gland: Studies of Seventy Autopsied Cases. The Journal of Clinical Endocrinology and Metabolism, Vol. 46, No. 6, (June, 1978), pp. (859-862), doi: 10.1210/jcem-46-6-859.

Zois, C., Stavrou, I., Kaiogera, C., Svarna, E., Dimolitais, I., Seferiadis, K., Tsatsoulis, A. (2003). High Prevalence of Autoimmune Thyroiditis in Schoolchildren after Elimination of Iodine Deficiency in Northwestern Greece. Thyroid, Vol. 13, No. 5, (2003), pp. (485-489), doi: 10.1089/105072503322021151. 


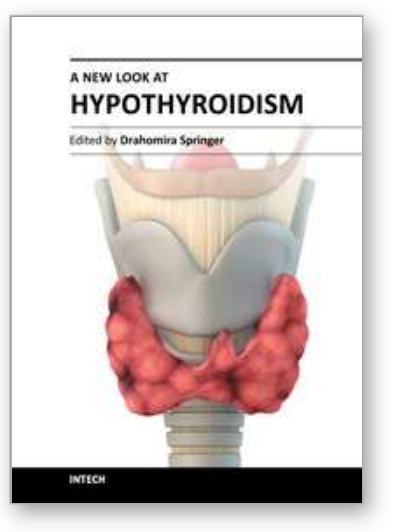

\author{
A New Look at Hypothyroidism \\ Edited by Dr. Drahomira Springer
}

ISBN 978-953-51-0020-1

Hard cover, 256 pages

Publisher InTech

Published online 17, February, 2012

Published in print edition February, 2012

Hypothyroidism is the most common thyroid disorder. It can cause a variety of changes in women's menstrual periods, reduce their chances of becoming pregnant, as well as affect both the course of pregnancy and the neuropsychological development of babies. During pregnancy there is a substantially increased need for thyroid hormones and a substantial risk that a previously unnoticed, subclinical or latent hypothyroidism will turn into overt hypothyroidism. The thyroid inflammation caused by the patient's own immune system may form autoimmune thyroiditis (Hashimoto's thyroiditis). Congenital hypothyroidism $(\mathrm{CH})$ occurs in approximately $1: 2,000$ to $1: 4,000$ newborns. Nearly all of the developed world countries currently practice newborn screening to detect and treat congenital hypothyroidism in the first weeks of life. "A New Look at Hypothyroidism" contains many important specifications and innovations for endocrine practice.

\title{
How to reference
}

In order to correctly reference this scholarly work, feel free to copy and paste the following:

Arvin Parvathaneni, Daniel Fischman and Pramil Cheriyath (2012). Hashimoto's Thyroiditis, A New Look at Hypothyroidism, Dr. Drahomira Springer (Ed.), ISBN: 978-953-51-0020-1, InTech, Available from: http://www.intechopen.com/books/a-new-look-at-hypothyroidism/hashimoto-s-disease

\section{INTECH}

open science | open minds

\section{InTech Europe}

University Campus STeP Ri

Slavka Krautzeka 83/A

51000 Rijeka, Croatia

Phone: +385 (51) 770447

Fax: +385 (51) 686166

www.intechopen.com

\section{InTech China}

Unit 405, Office Block, Hotel Equatorial Shanghai

No.65, Yan An Road (West), Shanghai, 200040, China

中国上海市延安西路65号上海国际贵都大饭店办公楼405单元

Phone: +86-21-62489820

Fax: +86-21-62489821 
(C) 2012 The Author(s). Licensee IntechOpen. This is an open access article distributed under the terms of the Creative Commons Attribution 3.0 License, which permits unrestricted use, distribution, and reproduction in any medium, provided the original work is properly cited. 